in atherosclerosis and type 2 diabetes. Sci Transl Med. 2011;3(81):81ps17.

11. Larsen CM, et al. Interleukin-1-receptor antagonist in type 2 diabetes mellitus. $N$ Engl J Med 2007;356(15):1517-1526.

12. Larsen CM, Faulenbach M, Vaag A, Ehses JA, Donath MY, Mandrup-Poulsen T. Sustained effects of interleukin-1 receptor antagonist treatment in type 2 diabetes. Diabetes Care. 2009;32(9):1663-1668.

13. Fearon WF, Fearon DT. Inflammation and cardiovascular disease: role of the interleukin-1 receptor antagonist. Circulation. 2008;117(20):2577-2579.

14. Duewell P, et al. NLRP3 inflammasomes are required for atherogenesis and activated by cholesterol crystals. Nature. 2010;464(7293):1357-1361.

15. Rajamaki K, et al. Cholesterol crystals activate the NLRP3 inflammasome in human macrophages: a novel link between cholesterol metabolism and inflammation. PLoS One. 2010;5(7):e11765.

16. Hansson GK, Klareskog L. Pulling down the plug on atherosclerosis: cooling down the inflammasome. Nat Med. 2011;17(7):790-791.

17. De Nardo D, Latz E. NLRP3 inflammasomes link inflammation and metabolic disease. Trends Immunol. 2011;32(8):373-379.

18. Kirii H, et al. Lack of interleukin-1beta decreases the severity of atherosclerosis in ApoE-deficient mice. Arterioscler Thromb Vasc Biol. 2003; 23(4):656-660

19. Bhaskar V, et al. Monoclonal antibodies targeting IL-1 beta reduce biomarkers of atherosclerosis in vitro and inhibit atherosclerotic plaque formation in Apolipoprotein E-deficient mice. Atherosclerosis. 2011;216(2):313-320
20. Ridker PM, Thuren T, Zalewski A, Libby P. Interleukin-1beta inhibition and the prevention of recurrent cardiovascular events: rationale and design of the Canakinumab Anti-inflammatory Thrombosis Outcomes Study (CANTOS). Am Heart J. 2011;162(4):597-605.

21. Alexander MR, et al. Genetic inactivation of IL-1 signaling enhances atherosclerotic plaque instability and reduces outward vessel remodeling in advanced atherosclerosis in mice. J Clin Invest. 2012; 122(1):70-79.

22. Chi $\mathrm{H}$, Messas E, Levine RA, Graves DT, Amar S Interleukin-1 receptor signaling mediates atherosclerosis associated with bacterial exposure and/or a high-fat diet in a murine apolipoprotein $\mathrm{E}$ heterozygote model: pharmacotherapeutic implications. Circulation. 2004;110(12):1678-1685

\title{
Redox redux: protecting the ischemic myocardium
}

\section{Oded N. Spindel and Bradford C. Berk}

Department of Medicine and Department of Pharmacology and Physiology, University of Rochester School of Medicine and Dentistry, Aab Cardiovascular Research Institute, Rochester, New York, USA.

\begin{abstract}
Cardiac ischemia-reperfusion (I-R) injury occurs upon prompt restoration of blood flow to the ischemic myocardium after an acute myocardial infarction. Interestingly, many of the features of I-R injury are related to impaired mitochondrial signaling and mitochondrial dysfunction. Restoring cardiac energy bioavailability and reduction-oxidation (redox) signaling are therefore important in recovery after I-R injury. In this issue of the $J C I$, Yoshioka and colleagues describe an important and unexpected role for thioredoxin-interacting protein (TXNIP) in the control of mitochondrial respiration and cell energy metabolism. Their findings could open the door for development of TXNIP-targeted therapeutic approaches for the treatment of cardiac I-R injury.
\end{abstract}

After acute myocardial infarction, prompt restoration of blood flow to the ischemic myocardium - through the use of thrombolytic therapy or primary percutaneous coronary intervention - limits infarct size and reduces mortality. However, the restoration of blood flow can result in additional myocardial damage, a phenomenon referred to as ischemiareperfusion (I-R) injury. Despite many promising preclinical approaches to improve cardiac function after I-R, their clinical translation has thus far yielded little therapeutic benefit (1). Emerging data suggest that thioredoxin-interacting protein (TXNIP) could provide a new candidate therapeutic target $(2,3)$, and a clear rationale for this is now provided by the work of Yoshioka et al. reported in this issue of the JCI (4).

Conflict of interest: Bradford C. Berk is on the Scientific Advisory Board for Merck.

Citation for this article: J Clin Invest. 2012; 122(1):30-32. doi:10.1172/JCI61467.
TXNIP controls energy bioavailability by altering mitochondrial redox state and respiration

Many of the features of I-R injury are related to mitochondrial dysfunction manifest by the uncoupling of oxidation-phosphorylation (OXPHOS) that leads to decreased ATP production and increased ROS generation (5). Restoring cardiac energy bioavailability and homeostatic reductionoxidation (redox) signaling are therefore important in recovery from I-R. Consequently, detailed understanding of the regulation of mitochondrial homeostasis and cellular bioenergetics is key to designing therapeutic approaches to improve cardiac function after I-R.

It has recently become clear that TXNIP plays a critical role in regulating mitochondrial homeostasis and cellular bioenergetics (6-8). TXNIP is the endogenous inhibitor of the two isoforms of thioredoxin, cytosolic thioredoxin (TRX1) and mitochondrial thioredoxin (TRX2), which are key regulators of cellular redox state insert (9). Because TXNIP inhibits TRX2 in the mitochondria, and mitochondrial function is critical to recovery from I-R injury, it is logical to believe that decreasing TXNIP expression should improve mitochondrial function and recovery from I-R injury.

This hypothesis is supported by recent data showing that TXNIP has redoxdependent effects on mitochondria (Figure 1 and refs. 7,8). One set of data indicates that TXNIP-deficient mice exhibit increased glycolysis and Akt signaling associated with impaired mitochondrial fuel oxidation and loss of phosphatase and tensin homolog (PTEN) activity (7). Mechanistically, the PTEN active site contains two critical cysteine residues (Cys-71 and Cys-124) that must be in the reduced form for proper catalytic activity. Alterations in the redox state of those residues are linked to the mitochondrial respiration rate and to the ability of TRX to interact with PTEN, which is regulated by TXNIP. The second set of data indicates that TXNIP translocates from the nucleus to the mitochondria in response to changes in cellular redox state and targets TRX2 (8). As a result, TRX2 activity decreases, which induces dissociation of TRX2 from apoptosis signal regulating kinase 1 (ASK1) and release of its inhibitory effect. ASK1 dissociation from TRX2 leads to increased ASK1 activity and subsequent induction of mitochondria-mediated apoptotic signaling, including cytochrome $c$ release and caspase- 3 activation. 


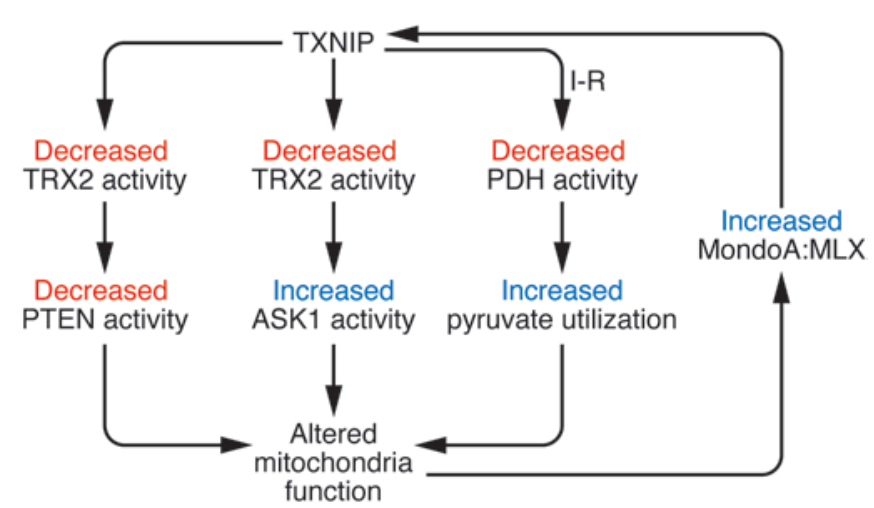

\section{Figure 1}

TXNIP regulates mitochondrial function via several pathways. First, TXNIP regulates fuel use in the mitochondria via inhibition of TRX2 and alteration of PTEN-Akt signaling. Second, TXNIP translocates to the mitochondria in response to changes in cellular redox state, resulting in inhibition of TRX2 and subsequent activation of ASK1 that leads to opening of the mitochondrial permeability transition pore. Third, as shown by Yoshioka et al., TXNIP interacts with PDH and acts as a metabolic switch between aerobic and anaerobic metabolism (4). Last, TXNIP expression is closely regulated by the MondoA:MLX transcription factor, which is activated by glucose uptake, glycolytic intermediates generated by the mitochondria, and lactate.

\section{An exciting and surprising insight into TXNIP control of mitochondria function}

Yoshioka et al. definitively prove the hypothesis that decreasing TXNIP expression improves recovery after I-R injury (4). Using mice in which Txnip was deleted in every cell, as well as those in which the gene was deleted in a cardiac-specific manner, the authors showed that after I-R injury, left ventricular function was improved in TXNIP-deficient versus wild-type mice. Unexpectedly, the improvement in left ventricular function was accompanied by a decrease, not an increase, in mitochondrial respiratory function. Yoshioka et al. went on to determine the mechanism underlying the beneficial effect of TXNIP deletion by several approaches. First, they performed bioinformatics analyses of the transcriptome and proteome in TXNIPdeficient mouse hearts to identify pathways altered by Txnip deletion. The mRNA expression approach revealed 390 genes regulated by TXNIP, of which 306 were downregulated. In addition, they showed an altered gene expression that primarily led to decreased expression of proteins important for energy metabolism. More specifically, proteins that are involved in OXPHOS or fatty acid/ carbohydrate metabolism associated with metabolic pathways in the mitochondria. The proteomics approach revealed 21 proteins that were differentially regulated, of which 13 had described cellular functions, and all were decreased in TXNIP-deficient mouse hearts. Among the 13 proteins, 12 were related to mitochondrial metabolism, which supports the notion that TXNIP is a key regulator of mitochondrial function in the heart.

Second, Yoshioka et al. used skinned cardiac myocyte fibers and isolated mitochondria to show that deletion of Txnip in mice was associated with reduced mitochondrial function. This was not due to a loss of respiratory complexes, but resulted from a functional loss of ADP-stimulated respiration. There was also no change in mitochondrial permeability transition pore opening. Third, the authors showed that, surprisingly, there was no change in the number of mitochondria or in their structure. However, electron microscopy showed large perimitochondrial lipid droplets and matrix granules in the mitochondria of TXNIP-deficient hearts, suggestive of altered fatty acid and lipid metabolism. Fourth, they measured the activity of TRX1 and TRX2. Although there was no change in TRX1 activity, there was a substantial increase in TRX2 activity and a decrease in ROS generation in TXNIP-deficient mice that was apparent after I-R injury. Fifth, they determined cellular ATP content after I-R, which was 2-fold greater in TXNIP-KO hearts. This was due to a shift from aerobic to anaerobic metabolism, as shown by blocking glycolysis. Finally, the authors studied the role of the pyruvate dehydrogenase complex, which regulates glycolytic

flux relative to mitochondrial respiration. They found that TXNIP binds to pyruvate dehydrogenase (PDH), specifically pyruvate dehydrogenase E1 component, subunit $\alpha$ (Figure 1). In TXNIP-deficient hearts, PDH activity was inhibited, and there was a shift in the use of glycolytically derived pyruvate away from its metabolism in mitochondria toward cytosolic lactate production and ATP generation.

In summary, Yoshioka et al. demonstrated that TXNIP acts as a metabolic switch to enhance ATP production by anaerobic pathways via its ability to inhibit mitochondrial respiration (4). Moreover, TXNIP was shown to mediate this function by both decreasing expression of mitochondrial OXPHOS proteins and by redirecting pyruvate away from mitochondria.

\section{Exciting findings, further complexity in TXNIP biology}

A complexity in interpreting the results of Yoshioka et al. is the fact that although TXNIP regulates mitochondrial respira-

Table 1

Functions of TXNIP in distinct subcellular compartments

\section{Function/effect \\ Nucleus}

Induces growth arrest

Regulates expression of hypertrophic genes

Induces expression of inflammatory genes

Cytosol

Inhibits TRX1

Degrades HIF1 $\alpha$

Activates ASK1

Mitochondria

Inhibits TRX2 and activates ASK1

Inhibits PTEN

Regulates aerobic/anaerobic respiration

Plasma membrane

Regulates tyrosine kinase receptor activation

Facilitates cargo transport
Reference

11

12

21

2, 22

13

23

8

7

4

15 
tion and energy production, TXNIP expression itself is regulated by glucose and glycolytic intermediates (6). It is therefore possible that drugs that inhibit TXNIP expression or function may paradoxically stimulate pathways that increase TXNIP expression (Figure 1). In addition, TXNIP appears to have many functions in subcellular compartments besides mitochondria (Table 1 and reviewed in ref. 10). First, in the nucleus, TXNIP acts as a growth suppressor via interaction and inhibition of Jun activation domain-binding protein 1 (JAB1) (11). TXNIP has also been shown to mediate TRX1 translocation into the nucleus, resulting in the regulation of cardiac hypertrophy (12). Second, TXNIP shuttles HIF $1 \alpha$ from the nucleus to the cytosol, targeting it for proteasomal degradation (13). Third, the TXNIP-TRX1 complex has previously been shown to translocate to the plasma membrane to promote activation of VEGFR2, resulting in endothelial cell survival and migration (14). Finally, TXNIP can facilitate the transport of proteins to the plasma membrane, an effect that may be important for cell signaling (15). These findings suggest multiple regulatory mechanisms for both TXNIP expression and TXNIP subcellular location, which together determine the overall function of TXNIP.

\section{Starting to unfold the complexity: TXNIP as an arrestin protein}

Another complexity in TXNIP biology is that it belongs to a class of proteins termed the $\alpha$-arrestins. The function of $\alpha$-arrestins is not well known, but it is clear that they participate in multiple protein interactions (16). The function of TXNIP as an arrestin is best exemplified by recent data demonstrating that the TXNIP-TRX1/2 complex is itself a signaling mediator $(14,17)$. With regard to this point, a protein-protein interactome analysis performed by Yoshioka et al. revealed two networks of TXNIP-interacting proteins that are candidates for future exploration of the arrestin role of TXNIP, which may or may not be related to its function in the mitochondria (4). The first network of TXNIP-interacting proteins identified by the authors involved retinoid $X$ receptor- $\alpha$ (RXRA) and its target, PPAR $\gamma$ coactivator $1-\alpha$ (PGC1 $\alpha)$. The second network involved p38 MAPK and
PGC1ß. It is likely that TXNIP, by interacting with proteins in these networks, regulates multiple cellular responses. Importantly, several of these proteins are regulators of mitochondrial metabolism, mitochondrial biogenesis, and cell metabolism (especially in adipocytes). It is not unexpected that TXNIP would be able to interact with a large network of proteins, since it contains several SH3 and PPxY motifs that can facilitate protein-protein interactions.

\section{Summary}

There is increasing appreciation of the importance of TXNIP in the cardiovascular system, since it has the ability to integrate signals related to changes in redox state, inflammation, metabolism, and biomechanical forces $(2,3,18-20)$. Furthermore, because TXNIP expression is highly regulated by multiple stimuli and has a very short half-life, it should be an easy target for drugs that limit its expression. Alternatively, drugs that prevent its interaction with key proteins, such as TRX1, TRX2, PDH, and nuclear transcription factors, should have powerful effects in a variety of diseases. The work by Yoshioka et al. (4) provides a further rationale to focus on TXNIP as a therapeutic target for I-R injury.

\section{Acknowledgments}

This work was supported by NIH grant HL 106158 to B.C. Berk.

Address correspondence to: Bradford C. Berk, Aab Cardiovascular Research Institute, University of Rochester, Box CVRI, 601 Elmwood Ave., Rochester, New York 14642, USA. Phone: 585.275.3407; Fax: 585.273.1059; E-mail: Bradford_berk@ urmc.rochester.edu.

1. Yellon DM, Hausenloy DJ. Myocardial reperfusion injury. N Engl J Med. 2007;357(11):1121-1135.

2. Yamawaki H, Pan S, Lee RT, Berk BC. Fluid shear stress inhibits vascular inflammation by decreasing thioredoxin-interacting protein in endothelial cells. J Clin Invest. 2005;115(3):733-738.

3. Wang Y, De Keulenaer GW, Lee RT. Vitamin D(3)up-regulated protein-1 is a stress-responsive gene that regulates cardiomyocyte viability through interaction with thioredoxin. J Biol Chem. 2002; 277(29):26496-26500.

4. Yoshioka J, et al. Deletion of thioredoxin-interacting protein in mice impairs mitochondrial function but protects the myocardium from ischemia-reperfusion injury. J Clin Invest. 2012; 122(1):267-279.

5. Di Lisa F, Bernardi P. Mitochondria and ischemia- reperfusion injury of the heart: fixing a hole. Cardiovasc Res. 2006;70(2):191-199.

6. Yu FX, Chai TF, He H, Hagen T, Luo Y. Thioredoxininteracting protein (Txnip) gene expression: sensing oxidative phosphorylation status and glycolytic rate. J Biol Chem. 2010;285(33):25822-25830.

7. Hui ST, et al. Txnip balances metabolic and growth signaling via PTEN disulfide reduction. Proc Natl Acad Sci US A. 2008;105(10):3921-3926.

8. Saxena G, Chen J, Shalev A. Intracellular shuttling and mitochondrial function of thioredoxin-interacting protein. J Biol Chem. 2010;285(6):3997-4005.

9. World CJ, Yamawaki H, Berk BC. Thioredoxin in the cardiovascular system. J Mol Med. 2006; 84(12):997-1003.

10. Spindel ON, World C, Berk B. Thioredoxin Interacting Protein (TXNIP): Redox dependent and independent regulatory mechanisms [published online ahead of print September 19, 2011]. Antioxid Redox Signal. doi:10.1089/ars.2011.4137.

11. Jeon JH, Lee KN, Hwang CY, Kwon KS, You KH, Choi I. Tumor suppressor VDUP1 increases p27(kip1) stability by inhibiting JAB1. Cancer Res. 2005; 65(11):4485-4489.

12. Ago T, et al. A redox-dependent pathway for regulating class II HDACs and cardiac hypertrophy. Cell. 2008; 133(6):978-993.

13. Shin D, et al. VDUP1 mediates nuclear export of HIF1alpha via CRM1-dependent pathway. Biochim Biophys Acta. 2008;1783(5):838-848.

14. World C, Spindel ON, Berk BC. Thioredoxin-interacting protein mediates TRX1 translocation to the plasma membrane in response to tumor necrosis factor- $\alpha$ : a key mechanism for vascular endothelial growth factor receptor- 2 transactivation by reactive oxygen species. Arterioscler Thromb Vasc Biol. 2011; 31(8):1890-1897.

15. Rauch S, Martin-Serrano J. Multiple interactions between the ESCRT machinery and arrestin-related proteins: implications for PPXY-dependent budding. J Virol. 2011;85(7):3546-3556.

16. Alvarez CE. On the origins of arrestin and rhodopsin. BMC Evol Biol. 2008;8:222.

17. Chutkow WA, Lee RT. Thioredoxin regulates adipogenesis through thioredoxin-interacting protein (Txnip) protein stability. J Biol Chem. 2011; 286(33):29139-29145.

18. Zhou R, Tardivel A, Thorens B, Choi I, Tschopp J. Thioredoxin-interacting protein links oxidative stress to inflammasome activation. Nat Immunol. 2010; 11(2):136-140

19. Chen J, Saxena G, Mungrue IN, Lusis AJ, Shalev A. Thioredoxin-interacting protein: a critical link between glucose toxicity and beta-cell apoptosis. Diabetes. 2008;57(4):938-944.

20. Schulze PC, Yoshioka J, Takahashi T, He Z, King GL, Lee RT. Hyperglycemia promotes oxidative stress through inhibition of thioredoxin function by thioredoxin-interacting protein. J Biol Chem. 2004; 279(29):30369-30374.

21. Perrone L, Devi TS, Hosoya K, Terasaki T, Singh LP. Thioredoxin interacting protein (TXNIP) induces inflammation through chromatin modification in retinal capillary endothelial cells under diabetic conditions. J Cell Physiol. 2009;221(1):262-272.

22. Li X, et al. Up-regulation of thioredoxin interacting protein (Txnip) by p38 MAPK and FOXO1 contributes to the impaired thioredoxin activity and increased ROS in glucose-treated endothelial cells. Biochem Biophys Res Commun. 2009;381(4):660-665.

23. Chen CL, Lin CF, Chang WT, Huang WC, Teng $\mathrm{CF}$, Lin YS. Ceramide induces p38 MAPK and JNK activation through a mechanism involving a thioredoxin-interacting protein-mediated pathway. Blood. 2008;111(8):4365-4374 